\title{
On $k$-Walk-Regular Graphs *
}

\author{
C. Dalfó, M.A. Fiol, E. Garriga \\ Departament de Matemàtica Aplicada IV \\ Universitat Politècnica de Catalunya \\ Barcelona, Catalonia (Spain) \\ $\{$ cdalfo,fiol, egarriga\}@ma4.upc.edu
}

Submitted: Feb 24, 2009; Accepted: Apr 8, 2009; Published: Apr 22, 2009

Mathematics Subject Classifications: 05C50, 05E30, 05C12, 05E35

\begin{abstract}
Considering a connected graph $G$ with diameter $D$, we say that it is $k$-walkregular, for a given integer $k(0 \leq k \leq D)$, if the number of walks of length $\ell$ between any pair of vertices only depends on the distance between them, provided that this distance does not exceed $k$. Thus, for $k=0$, this definition coincides with that of walk-regular graph, where the number of cycles of length $\ell$ rooted at a given vertex is a constant through all the graph. In the other extreme, for $k=D$, we get one of the possible definitions for a graph to be distance-regular. In this paper we show some algebraic characterizations of $k$-walk-regularity, which are based on the so-called local spectrum and predistance polynomials of $G$.
\end{abstract}

\section{Introduction}

Distance-regular graphs with diameter $D$ can be characterized by the invariance of the number of walks of length $\ell \geq 0$ between vertices at a given distance $i, 0 \leq i \leq D$ (see e.g. Rowlinson [11]). Similarly, walk-regular graphs are characterized by the fact that the number of closed walks of length $\ell \geq 0$ rooted at any given vertex is a constant (see e.g. Godsil [8]). Based on these definitions, in this paper we introduce a generalization of both distance-regularity and walk-regularity, which we call $k$-walk-regularity. In particular, we present some algebraic characterizations of $k$-walk-regular graphs in terms of the so-called local spectrum, which gives information of the graph when it is seen from a vertex, and the predistance polynomials of $G$.

${ }^{*}$ Research supported by the Ministerio de Educación y Ciencia, Spain, and the European Regional Development Fund under project MTM2008-06620-C03-01 and by the Catalan Research Council under project 2005SGR00256. 
We begin with some notation and basic results. Throughout this paper, $G=(V, E)$ denotes a simple, connected graph, with order $n=|V|$ and adjacency matrix $\boldsymbol{A}$. The distance between two vertices $u$ and $v$ is denoted by $\operatorname{dist}(u, v)$, so that the eccentricity of a vertex is $\operatorname{ecc}(u)=\max _{v \in V} \operatorname{dist}(u, v)$ and the diameter of the graph is $D=D(G)=$ $\max _{u \in V} \operatorname{ecc}(u)$. The spectrum of $G$ is denoted by

$$
\operatorname{sp} G=\operatorname{sp} \boldsymbol{A}=\left\{\lambda_{0}^{m_{0}}, \lambda_{1}^{m_{1}}, \ldots, \lambda_{d}^{m_{d}}\right\}
$$

with different eigenvalues of $G$ in decreasing order $\lambda_{0}>\lambda_{1}>\cdots>\lambda_{d}$ and the superscripts stand for their multiplicities $m_{i}=m\left(\lambda_{i}\right)$. In particular, note that $m_{0}=1$ (since $G$ is connected) and $m_{0}+m_{1}+\cdots+m_{d}=n$. It is well-known that the diameter of $G$ satisfies $D \leq d$ (see, for instance, Biggs [1]). Then, a graph with $D=d$ is said to have spectrally maximum diameter. This assures the existence of two vertices at (spectrally maximum) distance $d$. For a given ordering of the vertices of $G$, the vector space of linear combinations (with real coefficients) of the vertices is identified with $\mathbb{R}^{n}$, with canonical basis $\left\{\boldsymbol{e}_{u}: u \in V\right\}$. Let $Z=\prod_{i=0}^{d}\left(x-\lambda_{i}\right)$ be the minimal polynomial of $\boldsymbol{A}$. The vector space $\mathbb{R}_{d}[x]$ of real polynomials of degree at most $d$ is isomorphic to $\mathbb{R}[x] /(Z)$, and each polynomial $p \in \mathbb{R}_{d}[x]$ operates on the vector $\boldsymbol{w} \in \mathbb{R}^{n}$ by $p(\boldsymbol{A}) \boldsymbol{w}$. For every $0 \leq k \leq d$, the orthogonal projection of $\mathbb{R}^{n}$ onto $\mathcal{E}_{k}=\operatorname{Ker}\left(\boldsymbol{A}-\lambda_{k} \boldsymbol{I}\right)$ is given by the polynomial of degree $d$

$$
P_{k}=\frac{1}{\phi_{k}} \prod_{\substack{i=0 \\ i \neq k}}^{d}\left(x-\lambda_{i}\right)=\frac{(-1)^{k}}{\pi_{k}} \prod_{\substack{i=0 \\ i \neq k}}^{d}\left(x-\lambda_{i}\right),
$$

where $\phi_{k}=\prod_{i=0, i \neq k}^{d}\left(\lambda_{k}-\lambda_{i}\right)$ and $\pi_{k}=\left|\phi_{k}\right|$ are 'moment-like' parameters satisfying

$$
\sum_{k=0}^{d}(-1)^{k} \frac{\lambda_{k}^{\ell}}{\pi_{k}}= \begin{cases}0 & \text { if } 0 \leq \ell<d \\ 1 & \text { if } \ell=d\end{cases}
$$

(just express $x^{k}$ in terms of the basis $\left\{P_{0}, P_{1}, \ldots, P_{d}\right\}$ and equate coefficients of degree $d$ ). The matrices $\boldsymbol{E}_{k}=P_{k}(\boldsymbol{A})$ corresponding to these orthogonal projections are called the (principal) idempotents of $\boldsymbol{A}$. Then, the orthogonal decomposition of the unitary vector $\boldsymbol{e}_{u}$, representing vertex $u$, is:

$$
\boldsymbol{e}_{u}=\boldsymbol{z}_{u}^{0}+\boldsymbol{z}_{u}^{1}+\cdots+\boldsymbol{z}_{u}^{d}, \quad \text { where } \quad \boldsymbol{z}_{u}^{k}=P_{k}(\boldsymbol{A}) \boldsymbol{e}_{u}=\boldsymbol{E}_{k} \boldsymbol{e}_{u} \in \mathcal{E}_{k} .
$$

In particular, if $\boldsymbol{\nu}=\left(\nu_{u}\right)_{u \in V}$ is an eigenvector of $\lambda_{0}$, then $\boldsymbol{z}_{u}^{0}=\frac{\left(\boldsymbol{e}_{u}, \boldsymbol{\nu}\right)}{\|\boldsymbol{\nu}\|^{2}} \boldsymbol{\nu}=\frac{\nu_{u}}{\|\boldsymbol{\nu}\|^{2}} \boldsymbol{\nu}$, where $(\cdot, \cdot)$ stands for the standard Euclidean inner product.

The idempotents of $\boldsymbol{A}$ satisfy the following properties:

(a.1) $\boldsymbol{E}_{k} \boldsymbol{E}_{h}= \begin{cases}\boldsymbol{E}_{k} & \text { if } k=h, \\ \mathbf{0} & \text { otherwise }\end{cases}$

(a.2) $\boldsymbol{A} \boldsymbol{E}_{k}=\lambda_{k} \boldsymbol{E}_{k}$ 
(a.3) $p(\boldsymbol{A})=\sum_{k=0}^{d} p\left(\lambda_{k}\right) \boldsymbol{E}_{k}$, for any polynomial $p \in \mathbb{R}[x]$.

In particular, taking $p=1$ in (a.3), we have $\boldsymbol{E}_{0}+\boldsymbol{E}_{1}+\cdots+\boldsymbol{E}_{d}=\boldsymbol{I}$ (as expected, since the sum of all orthogonal projections gives the original vector, see e.g. Godsil [8]). Moreover, taking $p=x^{\ell}$, each power of $\boldsymbol{A}$ can be expressed as a linear combination of the idempotents $\boldsymbol{E}_{k}$ :

$$
\boldsymbol{A}^{\ell}=\sum_{k=0}^{d} \lambda_{k}^{\ell} \boldsymbol{E}_{k}
$$

From the decomposition (1), the $u$-local multiplicity of eigenvalue $\lambda_{k}$ is defined as

$$
m_{u}\left(\lambda_{k}\right)=\left\|\boldsymbol{z}_{u}^{k}\right\|^{2}=\left(\boldsymbol{E}_{k} \boldsymbol{e}_{u}, \boldsymbol{E}_{k} \boldsymbol{e}_{u}\right)=\left(\boldsymbol{E}_{k} \boldsymbol{e}_{u}, \boldsymbol{e}_{u}\right)=\left(\boldsymbol{E}_{k}\right)_{u u},
$$

(see Fiol and Garriga [5]), satisfying $\sum_{k=0}^{d} m_{u}\left(\lambda_{k}\right)=1$ and $\sum_{u \in V} m_{u}\left(\lambda_{k}\right)=m_{k}, 0 \leq k \leq$ $d$.

In particular, we say that a (connected) graph $G$ is spectrally regular when, for any $k=0,1, \ldots, d$, the $u$-local multiplicity of $\lambda_{k}$ does not depend on the vertex $u$. Then, the above equations imply that the (standard) multiplicity "splits" equitably among the $n$ vertices, giving $m_{u}\left(\lambda_{k}\right)=\frac{m_{k}}{n}$. In particular, since $m_{u}\left(\lambda_{0}\right)=\left\|\boldsymbol{z}_{u}^{0}\right\|^{2}=\frac{\nu_{u}^{2}}{\|\boldsymbol{\nu}\|^{2}}$, the spectral regularity implies the regularity of the graph because, in this case, $m_{u}\left(\lambda_{0}\right)=\frac{1}{n}$ and $\nu_{u}=\frac{\|\boldsymbol{\nu}\|}{\sqrt{n}}$ for all $u$, so that $\lambda_{0}$ has a constant eigenvector, which is a characteristic property of regular graphs.

Let $a_{u}^{(\ell)}=\left(\boldsymbol{A}^{\ell}\right)_{u u}$ denote the number of closed walks of length $\ell$ rooted at vertex $u$. When the number $a_{u}^{(\ell)}$ only depends on $\ell$, in which case we write $a_{u}^{(\ell)}=a^{(\ell)}$, the graph $G$ is called walk-regular (a concept introduced by Godsil and McKay in [9]). Notice that, as $a_{u}^{(2)}=\delta_{u}$, the degree of vertex $u$, every walk-regular graph is also regular.

In the context of walk-regular graphs, the following result was given by Fiol and Garriga [4] and by Delorme and Tillich [3]:

Proposition 1.1 A connected graph $G$ is spectrally regular if and only if it is walk-regular.

Consequently, from now on we will indistinctly say that a graph $G$ is spectrally regular or that it is walk-regular.

\section{The predistance polynomials}

From the spectrum of a given graph sp $G=\left\{\lambda_{0}^{m_{0}}, \lambda_{1}^{m_{1}}, \ldots, \lambda_{d}^{m_{d}}\right\}$, we consider the following scalar product in $\mathbb{R}_{d}[x]$ :

$$
\langle p, q\rangle=\frac{1}{n} \operatorname{tr}(p(\boldsymbol{A}) q(\boldsymbol{A}))=\frac{1}{n} \sum_{k=0}^{d} m_{k} p\left(\lambda_{k}\right) q\left(\lambda_{k}\right) .
$$


Then, by using the Gram-Schmidt method and normalizing appropriately, it is immediate to prove the existence and uniqueness of an orthogonal system of polynomials $\left\{p_{k}\right\}_{0 \leq k \leq d}$ called predistance polynomials which, for any $0 \leq h, k \leq d$, satisfy:

(b.1) degree $\left(p_{k}\right)=k$;

$(b .2)\left\langle p_{h}, p_{k}\right\rangle=0$ if $h \neq k$;

(b.3) $\left\|p_{k}\right\|^{2}=p_{k}\left(\lambda_{0}\right)$.

Fiol and Garriga [5, 6] showed that such a system is unique and it is also characterized by any of the two following conditions:

(c.1) $p_{0}=1, \quad a_{k}+b_{k}+c_{k}=\lambda_{0}$ for $0 \leq k \leq d$, where $a_{k}, b_{k}$ and $c_{k}$ are the corresponding coefficients of the three-term recurrence

$$
x p_{k}=b_{k-1} p_{k-1}+a_{k} p_{k}+c_{k+1} p_{k+1} \quad(0 \leq k \leq d)
$$

(that is, the Fourier coefficients of $x p_{k}$ in terms of $p_{k-1}, p_{k}$, and $p_{k+1}$, respectively) initiated with $p_{-1}=0$ and $p_{0}$ any non-zero constant.

(c.2) $H=\sum_{k=0}^{d} p_{k}=\frac{n}{\pi_{0}} \prod_{k=1}^{d}\left(x-\lambda_{k}\right)=n P_{0}$.

The reader familiar with the theory of distance-regular graphs will have already noted that the predistance polynomials can be thought as a generalization of the so-called "distance polynomials". Recall that, in a distance-regular graph $G$, such polynomials satisfy

$$
p_{k}(\boldsymbol{A})=\boldsymbol{A}_{k} \quad(0 \leq k \leq d)
$$

where $\boldsymbol{A}_{k}$ stands for the adjacency matrix of the distance- $k$ graph $G_{k}$ (where two vertices $u$ and $v$ are adjacent if and only if $\operatorname{dist}(u, v)=k$ in $G)$, usually called the $k$-th distance matrix of $G$ (see, for instance, Brouwer, Cohen and Neumaier [2]). Also, recall that the polynomial $H$ in (c.2) is the Hoffman polynomial characterizing the regularity of $G$ by the condition $H(\boldsymbol{A})=\boldsymbol{J}$, the all-1 matrix (see Hoffman [10]).

In our context, the predistance polynomials allow us to give another characterization of walk-regularity (or spectral regularity), as it is shown in the following new result:

Proposition 2.1 Let $G$ be a (connected) graph with adjacency matrix $\boldsymbol{A}$ having $d+$ 1 distinct eigenvalues, and with predistance polynomials $p_{0}, p_{1}, \ldots, p_{d}$. Then, the two following statements are equivalent:

(a) $G$ is walk-regular.

(b) The matrices $p_{k}(\boldsymbol{A}), 1 \leq k \leq d$, have null diagonals. 
Proof. Assume first that (a) holds: if $G$ is walk-regular, then the diagonal vector of $\boldsymbol{A}^{\ell}$ is $\operatorname{diag}\left(\boldsymbol{A}^{\ell}\right)=a^{(\ell)} \boldsymbol{j}$, with $\boldsymbol{j}$ being the all-1 vector. Taking the set $\mathcal{C}=\left\{a^{(0)}, a^{(1)}, \ldots, a^{(d)}\right\}$, we introduce the following notation: Given a polynomial $p=\sum_{i=0}^{d} \alpha_{i} x^{i}$, let $p(\mathcal{C})=$ $\sum_{i=0}^{d} \alpha_{i} a^{(i)}$. Since $\left(p_{k}(\boldsymbol{A})\right)_{u u}=p_{k}(\mathcal{C})$ for every vertex $u, \operatorname{diag}\left(p_{k}(\boldsymbol{A})\right)=p_{k}(\mathcal{C}) \boldsymbol{j}$. But, for $1 \leq k \leq d$, we have

$$
0=\left\langle p_{k}, p_{0}\right\rangle=\frac{1}{n} \operatorname{tr}\left(p_{k}(\boldsymbol{A})\right)=p_{k}(\mathcal{C}),
$$

so that $\operatorname{diag}\left(p_{k}(\boldsymbol{A})\right)=\mathbf{0}$.

Now suppose that $(b)$ holds. Then, by using the expression

$$
x^{\ell}=\sum_{k=0}^{\ell} \alpha_{\ell k} p_{k},
$$

where $\alpha_{\ell k}$ are the Fourier coefficients of $x^{\ell}$ in terms of $p_{k}$, we have

$$
\operatorname{diag}\left(\boldsymbol{A}^{\ell}\right)=\sum_{k=0}^{\ell} \alpha_{\ell k} \operatorname{diag}\left(p_{k}(\boldsymbol{A})\right)=\alpha_{\ell 0} \boldsymbol{j} .
$$

Therefore, $a_{u}^{(\ell)}=\alpha_{\ell 0}$, which is independent of $u$ and the graph is walk-regular. (Notice that, since $p_{0}=1, \alpha_{\ell 0}=\frac{\left\langle x^{\ell}, 1\right\rangle}{\|1\|^{2}}=\frac{1}{n} \sum_{k=0}^{d} m_{k} \lambda_{k}^{\ell}$, as expected.)

Note that property $(b)$ is also satisfied in the case of distance-regularity, as $p_{k}(\boldsymbol{A})=\boldsymbol{A}_{k}$ and, for $k>0,\left(\boldsymbol{A}_{k}\right)_{u u}=\operatorname{dist}(u, u)=0$ for any vertex $u \in V$.

\section{$3 \quad k$-Walk-regular graphs}

The result given in Proposition 2.1 can be generalized if we consider the following new definition. Let $G$ be a (connected) graph with diameter $D$. For a given integer $k$, $0 \leq k \leq D$, we say that $G$ is $k$-walk-regular if the number of walks of length $\ell$ between vertices $u$ and $v$, that is, $a_{u v}^{(\ell)}=\left(\boldsymbol{A}^{\ell}\right)_{u v}$, only depends on the distance between $u$ and $v$, provided that $\operatorname{dist}(u, v)=i \leq k$. If this is the case, we write $a_{u v}^{(\ell)}=a_{i}^{(\ell)}$. Thus, a 0-walk-regular graph is the same concept as a walk-regular graph. In the other extreme, the distance-regular graphs correspond to the case of $D$-walk-regular graphs (see e.g. Rowlinson [11]). Note that, obviously, if $G$ is a $k$-walk-regular graph, then it is also $k^{\prime}$-walk-regular for any $k^{\prime} \leq k$. This is consequent with the fact that a distance-regular graph is also walk-regular. To illustrate our new definition, a family of graphs which are 1-walk-regular (but not $k$-walk-regular for $k>1$ ) are the Cartesian products of cycles $C_{m} \times C_{m}$ with $m \geq 5$. In fact, notice that all these graphs are vertex- and edge-transitive. For instance, $C_{5} \times C_{5}$ has diameter $D=2$, number of different eigenvalues $d+1=6$, and sets $\mathcal{C}=\left\{a_{0}^{(\ell)}\right\}_{0 \leq \ell \leq 5}=\{1,0,4,0,36,4\}$ and $\mathcal{W}=\left\{a_{1}^{(\ell)}\right\}_{0 \leq \ell \leq 5}=\{0,1,0,9,1,100\}$.

As in the case of walk-regularity, the concept of $k$-walk-regularity can also be seen as the invariance of some entries of the idempotents. By analogy with local multiplicities, which correspond to the diagonal of the matrix, Fiol, Garriga and Yebra [7] called these 
entries the crossed (uv-) local multiplicities of $\lambda_{h}$, and they were denoted by $m_{u v}\left(\lambda_{h}\right)$. In terms of the orthogonal projection of the canonical vectors $\boldsymbol{e}_{u}$, the crossed local multiplicities are obtained by the Euclidean products

$$
m_{u v}\left(\lambda_{h}\right)=\left(\boldsymbol{E}_{h}\right)_{u v}=\left(\boldsymbol{E}_{h} \boldsymbol{e}_{u}, \boldsymbol{e}_{v}\right)=\left(\boldsymbol{E}_{h} \boldsymbol{e}_{u}, \boldsymbol{E}_{h} \boldsymbol{e}_{v}\right)=\left(\boldsymbol{z}_{u}^{h}, \boldsymbol{z}_{v}^{h}\right) \quad(u, v \in V) .
$$

Now, for a given $k, 0 \leq k \leq d$, we say that graph $G$ is $k$-spectrally regular when, for any $h=0,1, \ldots, d$, the crossed $u v$-local multiplicities of $\lambda_{h}$ only depend on the distance between $u$ and $v$, provided that $i=\operatorname{dist}(u, v) \leq k$. In this case, we write $m_{u v}\left(\lambda_{h}\right)=m_{i h}$.

At this point, we are ready to give the following result (where "o" stands for the Schur or Hadamard - componentwise - product of matrices), relating the $k$-walk-regularity to the $k$-spectral regularity and the matrices obtained from the predistance polynomials. In the second case, these polynomials give the distance matrices, but only when we look through a 'window' defined by the matrix $\boldsymbol{S}_{k}=\boldsymbol{A}_{0}+\boldsymbol{A}_{1}+\cdots+\boldsymbol{A}_{k}$.

Theorem 3.1 Let $G$ be a graph with adjacency matrix $\boldsymbol{A}$ having $d+1$ distinct eigenvalues, and with predistance polynomials $p_{0}, p_{1}, \ldots, p_{d}$. Then, for a given integer $k, 0 \leq k \leq D$, the three following statements are equivalent:

(a) $G$ is k-walk-regular.

(b) $G$ is k-spectrally regular.

(c) $\boldsymbol{S}_{k} \circ p_{i}(\boldsymbol{A})=\boldsymbol{S}_{k} \circ \boldsymbol{A}_{i}$ for any $0 \leq i \leq d$.

Proof. $(a) \Leftrightarrow(b)$ : The equivalence between $(a)$ and $(b)$ is proved as follows: From Eq. (2), we now have that the number of walks $a_{u v}^{(\ell)}$ can be computed in terms of the crossed $u v$-local multiplicities as

$$
a_{u v}^{(\ell)}=\left(\boldsymbol{A}^{\ell}\right)_{u v}=\sum_{h=0}^{d} m_{u v}\left(\lambda_{h}\right) \lambda_{h}^{\ell} .
$$

Then, if $G$ is $k$-spectrally regular, this gives

$$
a_{u v}^{(\ell)}=\frac{1}{n} \sum_{k=0}^{d} m_{i h} \lambda_{h}^{\ell},
$$

for any $u, v \in V$ such that $\operatorname{dist}(u, v)=i \leq k$, and $\ell \geq 0$. Therefore, $a_{u v}^{(\ell)}$ is independent of $u, v$, provided that $\operatorname{dist}(u, v)=i \leq k$, and $G$ is $k$-walk-regular. Conversely, suppose that $G$ is $k$-walk-regular and consider the set of numbers of $(u, v)$-walks $\mathcal{W}=\left\{a_{i}^{(0)}, a_{i}^{(1)}, \ldots, a_{i}^{(d)}\right\}$, where $i=\operatorname{dist}(u, v) \leq k$. Now, given a polynomial $p=\sum_{j=0}^{d} \alpha_{j} x^{j}$, we define $p(\mathcal{W})=$ $\sum_{j=0}^{d} \alpha_{j} a_{i}^{(j)}$. Then, we can obtain the crossed $u v$-local multiplicities as

$$
m_{u v}\left(\lambda_{h}\right)=\left(\boldsymbol{E}_{h}\right)_{u v}=\left(P_{h}(\boldsymbol{A})\right)_{u v}=P_{h}(\mathcal{W}),
$$

which turn out to be independent of $u, v$ and $G$ is $k$-spectrally regular. 
$(a),(b) \Rightarrow(c)$ : We want to prove that $p_{i}(\boldsymbol{A})=\boldsymbol{A}_{i}$ if $i \leq k$ and, otherwise, $\boldsymbol{S}_{k} \circ p_{i}(\boldsymbol{A})=$ $\boldsymbol{O}$, the all-0 matrix . Then, if $G$ is $k$-walk-regular, there are constants $a_{i}^{(\ell)}$, for any $0 \leq i \leq k$ and $\ell \geq 0$ satisfying

$$
\boldsymbol{A}^{\ell}=\sum_{i=0}^{k} a_{i}^{(\ell)} \boldsymbol{A}_{i} \quad(\ell \leq k),
$$

where, clearly, $a_{i}^{(\ell)}=0$ when $\ell<i$. As a matrix equation (writing only the terms with $\ell \leq k$ ), we get

$$
\left(\begin{array}{c}
\boldsymbol{I} \\
\boldsymbol{A} \\
\boldsymbol{A}^{2} \\
\cdot \\
\cdot \\
\boldsymbol{A}^{k}
\end{array}\right)=\left(\begin{array}{cccccc}
a_{0}^{(0)} & & & & & \\
a_{0}^{(1)} & a_{1}^{(1)} & & & & \\
a_{0}^{(2)} & a_{1}^{(2)} & a_{2}^{(2)} & & & \\
\cdot & \cdot & \cdot & \cdot & & \\
\cdot & \cdot & \cdot & \cdot & \cdot & \\
a_{0}^{(k)} & a_{1}^{(k)} & \cdot & \cdot & \cdot & a_{k}^{(k)}
\end{array}\right)\left(\begin{array}{c}
\boldsymbol{I} \\
\boldsymbol{A} \\
\boldsymbol{A}_{2} \\
\cdot \\
\cdot \\
\boldsymbol{A}_{k}
\end{array}\right),
$$

where the lower triangular matrix $\boldsymbol{T}$, with rows and columns indexed with the integers $0,1 \ldots, k$, has entries $(\boldsymbol{T})_{\ell i}=a_{i}^{(\ell)}$. In particular, note that $a_{0}^{(0)}=a_{1}^{(1)}=1$ and $a_{0}^{(1)}=0$. Moreover, since $a_{i}^{(i)}>0$ for all $0 \leq i \leq k$, such a matrix has an inverse, which is also a lower triangular matrix, and hence each $\boldsymbol{A}_{i}$ is a polynomial, say $q_{i}$, of degree $i$ in $\boldsymbol{A}$. These polynomials are orthogonal with respect to the scalar product (3) since

$$
\left\langle q_{i}, q_{j}\right\rangle=\frac{1}{n} \operatorname{tr}\left(q_{i}(\boldsymbol{A}) q_{j}(\boldsymbol{A})\right)=\frac{1}{n} \operatorname{tr}\left(\boldsymbol{A}_{i} \boldsymbol{A}_{j}\right)=0 \quad(i \neq j) .
$$

Moreover, as $\boldsymbol{A}_{i} \boldsymbol{j}=q_{i}(\boldsymbol{A}) \boldsymbol{j}=q_{i}\left(\lambda_{0}\right) \boldsymbol{j}$, the number of vertices at distance $i, 0 \leq i \leq k$, from a given vertex $u$ is a constant through all the graph: $n_{i}=\left(\boldsymbol{A}_{i}^{2}\right)_{u u}=q_{i}\left(\lambda_{0}\right)$ for every $u \in V$. Thus,

$$
\left\|q_{i}\right\|^{2}=\frac{1}{n} \operatorname{tr}\left(q_{i}^{2}(\boldsymbol{A})\right)=\frac{1}{n} \operatorname{tr}\left(\boldsymbol{A}_{i}^{2}\right)=q_{i}\left(\lambda_{0}\right)
$$

and, therefore, the obtained polynomials are, in fact, the (pre)distance polynomials $q_{i}=$ $p_{i}, 0 \leq i \leq k$, as claimed. Let us now prove the second part of the statement: if $j>k$, then $p_{j}(\boldsymbol{A})_{u v}=0$ provided that $\operatorname{dist}(u, v) \leq k$. First, note that, from property $(a .2)$ of the idempotents, we have

$$
\left(p_{i}(\boldsymbol{A}) \boldsymbol{E}_{h}\right)_{u u}=p_{i}\left(\lambda_{h}\right)\left(\boldsymbol{E}_{h}\right)_{u u}=p_{i}\left(\lambda_{h}\right) m_{u}\left(\lambda_{h}\right)=p_{i}\left(\lambda_{h}\right) \frac{m_{h}}{n}
$$

for any $0 \leq i \leq k$ and $0 \leq h \leq d$. But, if $i=\operatorname{dist}(u, v) \leq k$, we already know that $p_{i}(\boldsymbol{A})=\boldsymbol{A}_{i}$ and then,

$$
\left(p_{i}(\boldsymbol{A}) \boldsymbol{E}_{h}\right)_{u u}=\left(\boldsymbol{A}_{i} \boldsymbol{E}_{h}\right)_{u u}=\sum_{v \in V}\left(\boldsymbol{A}_{i}\right)_{u v}\left(\boldsymbol{E}_{h}\right)_{u v}=\sum_{v \in \Gamma_{i}(u)} m_{u v}\left(\lambda_{h}\right)=n_{i} m_{i h},
$$


where we have used the invariance of the crossed local multiplicities, $m_{u v}\left(\lambda_{h}\right)=m_{i h}$, and the number of vertices at distance $i(\leq k)$ from any given vertex, $n_{i}=p_{i}\left(\lambda_{0}\right)$. Equating (5) and (6) we obtain:

$$
m_{i h}=\frac{m_{h} p_{i}\left(\lambda_{h}\right)}{n p_{i}\left(\lambda_{0}\right)} \quad(0 \leq i \leq k, 0 \leq h \leq d) .
$$

Using property (a.3) of the idempotents and the above values of the crossed multiplicities, we finally get:

$$
\begin{aligned}
p_{j}(\boldsymbol{A})_{u v} & =\sum_{h=0}^{d} p_{j}\left(\lambda_{h}\right)\left(\boldsymbol{E}_{h}\right)_{u v}=\sum_{h=0}^{d} p_{j}\left(\lambda_{h}\right) m_{i h} \\
& =\frac{1}{n p_{i}\left(\lambda_{0}\right)} \sum_{h=0}^{d} m_{h} p_{j}\left(\lambda_{h}\right) p_{i}\left(\lambda_{h}\right)=\frac{1}{p_{i}\left(\lambda_{0}\right)}\left\langle p_{j}, p_{i}\right\rangle=0 \quad(j>k \geq i) .
\end{aligned}
$$

$(c) \Rightarrow(b)$ : Conversely, assume that $(c)$ holds and, for every $h, 0 \leq h \leq d$, consider the expression of $P_{h}=\sum_{j=0}^{d} \beta_{h j} p_{j}$, where $\beta_{h j}$ is the Fourier coefficient of $P_{h}$ in terms of $p_{j}$. Then, if $\operatorname{dist}(u, v)=i \leq k$,

$$
m_{u v}\left(\lambda_{h}\right)=\left(\boldsymbol{E}_{h}\right)_{u v}=\sum_{j=0}^{d} \beta_{h j} p_{j}(\boldsymbol{A})_{u v}=\sum_{j=0}^{k} \beta_{h j}\left(\boldsymbol{A}_{j}\right)_{u v}+\sum_{j=k+1}^{d} \beta_{h j}\left(p_{j}(\boldsymbol{A})\right)_{u v}=\beta_{h i}
$$

Consequently, the crossed local multiplicities $m_{u v}\left(\lambda_{h}\right)=\beta_{h i}$ only depend on the distance $\operatorname{dist}(u, v)=i$, and $G$ is $k$-spectrally regular. (Notice that, $\beta_{h i}=m_{i h}=\frac{\left\langle P_{h}, p_{i}\right\rangle}{\left\|p_{i}\right\|^{2}}=$ $\frac{1}{p_{i}\left(\lambda_{0}\right) n} \sum_{j=0}^{d} m_{j} P_{h}\left(\lambda_{j}\right) p_{i}\left(\lambda_{j}\right)=\frac{m_{h} p_{i}\left(\lambda_{h}\right)}{n p_{i}\left(\lambda_{0}\right)}$, in concordance with $(7)$.)

Note that Propositions 1.1 and 2.1 can also be seen as corollaries of this theorem.

\section{References}

[1] N. Biggs, Algebraic Graph Theory, Cambridge University Press, Cambridge, 1974; second edition, 1993.

[2] A.E. Brouwer, A.M. Cohen, and A. Neumaier, Distance-Regular Graphs, SpringerVerlag, Berlin-New York, 1989.

[3] C. Delorme and J.P. Tillich, Eigenvalues, eigenspaces and distances to subsets, Discrete Math. 165/166 (1997) 161-184.

[4] M.A. Fiol and E. Garriga, The alternating and adjacency polynomials, and their relation with the spectra and diameters of graphs, Discrete Appl. Math. 87 (1998), no. $1-3,77-97$.

[5] M.A. Fiol and E. Garriga, From local adjacency polynomials to locally pseudodistance-regular graphs, J. Combin. Theory Ser. B 71 (1997) 162-183. 
[6] M.A. Fiol and E. Garriga, On the algebraic theory of pseudo-distance-regularity around a set, Linear Algebra Appl. 298 (1999) 115-141.

[7] M.A. Fiol, E. Garriga, and J.L.A. Yebra, Boundary graphs: The limit case of a spectral property, Discrete Math. 226 (2001), no. 1-3, 155-173.

[8] C.D. Godsil, Algebraic Combinatorics, Chapman and Hall, New York, 1993.

[9] C.D. Godsil and B.D. McKay, Feasibility conditions for the existence of walk-regular graphs, Linear Algebra Appl. 30 (1980) 51-61.

[10] A.J. Hoffman, On the polynomial of a graph, Amer. Math. Monthly 70 (1963) 30-36.

[11] P. Rowlinson, Linear algebra, in Graph Connections (ed. L.W. Beineke and R.J. Wilson), Oxford Lecture Ser. Math. Appl., Vol. 5, 86-99, Oxford Univ. Press, New York, 1997. 